Proyecciones Journal of Mathematics

Vol. 35, Nº 3, pp. 317-338, September 2016.

Universidad Católica del Norte

Antofagasta - Chile

\title{
Stability and boundedness in differential systems of third order with variable delay
}

\author{
Cemil Tunc \\ Yuzuncu University, Turkey \\ Received: April 2016. Accepted : June 2016
}

\begin{abstract}
In this paper, we consider a non-linear system of differential equations of third order with variable delay. We discuss the globally asymptotic stability/uniformly stability, boundedness and uniformly boundedness of solutions for the considered system. The technique of proofs involves defining an appropriate Lyapunov functional. The obtained results include and improve the results in literature.
\end{abstract}

Keywords : Globally asymptotic stability, boundedness, Lyapunov functional, delay, differential system, third order.

2010 Mathematics Subject Classifications : 34K12, 34K20. 


\section{Introduction}

In the last years, there is a good amount of interest in the qualitative behaviors of ordinary and functional differential equations of third order without and with delay, see the book of Reissig et al. [1] as a good survey for the works done by 1974 and the papers of Ademola and Arawomo ([2], [3], [4], [5]), Ademola et al. [6], Afuwape and Castellanos [7], Afuwape and Omeike [8], Ahmad and Rama Mohana Rao [9], Bai and Guo [10], Ezeilo [11], Ezeilo and Tejumola [12], Graef et al. ([13], [14]), Graef and Tunc [15], Korkmaz and Tunc [16], Mahmoud and Tunc [17], Ogundare [18], Ogundare et al. [19], Olutimo [20], Omeike [21], Qian [22], Remili and Oudjedi [23], Sadek [24], Swick [25], Tejumola and Tchegnani [26], Tunc ([27]-[44]), Tunc and Ates [45], Tunc and Gozen [46], Tunc and Mohammed [47], Tunc and Tunc [48], Zhang and $\mathrm{Yu}$ [49], Zhu [50] and theirs references. However, to the best of our knowledge from the literature, by this time, no attention was given to the investigation of the globally asymptotic stability/uniformly stability, boundedness and uniformly boundedness in the systems of nonlinear functional differential equations of third order with variable delay, except the recent work of Omeike [21].

Besides, it is well known that differential equations of third order play extremely important and useful roles in many scientific areas such as atomic energy, biology, chemistry, control theory, economy, engineering, information theory, mechanics, medicine, physics, etc.. Indeed, we can find applications such as nonlinear oscillations in Afuwape et al. [51], Andres [52], Fridedrichs [53], physical applications in Animalu and Ezeilo [54], non- resonant oscillations in Ezeilo and Onyia [55], prototypical examples of complex dynamical systems in a high-dimensional phase space, displacement in a mechanical system, velocity, acceleration in Chlouverakis and Sprott [56], Eichhorn et al. [57], Linz [58], the biological model and other models in Cronin-Scanlon [59], problems in biomathematics in Chlouverakis and Sprott [56], electronic theory in Rauch [60], and etc. Further, we refer the readers to the book of Smith [61] for some important applications of delay differential equation in sciences, biomathematics, engineering, and etc..

In 2015, Omeike [21] investigated the stability and boundedness of nonlinear differential system of third order with variable delay, $\tau(t)$ :

$$
X^{\prime \prime \prime}+A X^{\prime \prime}+B X^{\prime}+H(X(t-\tau(t)))=P(t) .
$$

In this paper, we consider nonlinear differential system of the third 
order with variable delay, $\tau(t)$ :

$$
X^{\prime \prime \prime}+A X^{\prime \prime}+G\left(X^{\prime}(t-\tau(t))\right)+H(X(t-\tau(t)))=F\left(t, X, X^{\prime}, X^{\prime \prime}\right),
$$

where $X \in \mathcal{R}^{n}, \mathrm{t} \in[0, \infty), \mathcal{R}^{+}=[0, \infty), \tau(t)$ is a continuous differentiable function such that $0 \leq \tau(t) \leq \tau_{0}$ is a positive constant and $\tau^{\prime}(t) \leq \tau_{1}$, $\left(0<\tau_{1}<1\right), A$ is an $n \times n$ - constant symmetric matrix, $G, H: \mathcal{R}^{n} \rightarrow \mathcal{R}^{n}$ are continuous differentiable functions with $G(0)=H(0)=0$ such that the Jacobian matrices $J_{G}\left(X^{\prime}\right)$ and $J_{H}(X)$ exist and are symmetric and continuous, that is,

$$
\mathrm{J}_{G}\left(X^{\prime}\right)=\left(\frac{\partial g_{i}}{\partial x_{j}^{\prime}}\right), J_{H}(X)=\left(\frac{\partial h_{i}}{\partial x_{j}}\right),(i, j=1,2, \ldots, n),
$$

exist and are symmetric and continuous, where $\left(x_{1}, x_{2}, \ldots, x_{n}\right),\left(x_{1}^{\prime}, x_{2}^{\prime}, \ldots, x_{n}^{\prime}\right)$, $\left(g_{i}\right)$ and $\left(h_{i}\right)$ are components of $X, X^{\prime}, G$ and $H$, respectively, $F: \mathcal{R}^{+} \times$ $\mathcal{R}^{n} \times \mathcal{R}^{n} \times \mathcal{R}^{n} \rightarrow \mathcal{R}^{n}$ is a continuous function and the primes in Eq. (1.2) indicate differentiation with respect to $t, t \geq t_{0} \geq 0$.

It is more convenient to consider not Eq. (1.2) itself, but rather the system

$$
\begin{aligned}
& X_{1}^{\prime}=X_{2}, X_{2}^{\prime}=X_{3} \\
& X_{3}^{\prime}=-A X_{3}-G\left(X_{2}\right)-H\left(X_{1}\right)+\int_{t-\tau(t)}^{t} J_{G}\left(X_{2}(s)\right) X_{3}(s) d s \\
& \quad+\int_{t-\tau(t)}^{t} J_{H}\left(X_{1}(s)\right) X_{2}(s) d s+F\left(t, X_{1}, X_{2}, X_{3}\right) .
\end{aligned}
$$

System (1.3) is obtained from Eq. (1.2) by setting $X=X_{1}, X^{\prime}=X_{2}$ and $X^{\prime \prime}=X_{3}$.

The continuity of the functions $\tau, H, G$ and $F$ is a sufficient condition to guarantee the existence of solutions of Eq. (1.2). Besides, we assume that the functions $H, G$ and $F$ satisfy a Lipschitz condition with respect to their respective arguments, like $X, X^{\prime}$ and $X^{\prime \prime}$. In this case, the uniqueness of solutions of Eq. (1.2) is guaranteed.

The motivation of this paper comes from the results established by Omeike [21], the mentioned books, papers and theirs references. The main 
purpose of this paper is to get new the globally asymptotic stability / uniformly stability, boundedness and uniformly boundedness results in Eq. (1.2) by defining a suitable new Lyapunov functional. By this paper, we extend and improve the stability and boundedness results of Omeike [21], and we give additional two results to that in Omeike [21], like uniformly stability and uniformly boundedness results. It follows that if we choose $G\left(X^{\prime}(t-\tau(t))\right)=B X^{\prime}(t), B$ is an $n \times n$-constant symmetric matrix, and $F\left(t, X, X^{\prime}, X^{\prime \prime}\right)=P(t)$ in Eq. (1.2), then Eq. (1.2) reduces to Eq. (1.1), which is discussed by Omeike [21]. This means that instead of the linear term $B X^{\prime}(t)$ in Eq. (1.1), we take the non-linear term $G\left(X^{\prime}(t-\tau(t))\right)$ which includes a variable delay $\tau(t)$, and we also take a nonlinear generalization of the term $P(t)$ in Eq. (1.1) like $F\left(t, X, X^{\prime}, X^{\prime \prime}\right)$. Probably, these cases seem as similarity of Eq. (1.1) and Eq. (1.2).

However, till now, throughout all the papers published in the literature, no author discussed the stability and boundedness of the solutions when we take the possible second term in Eq. (1.1), $G\left(X^{\prime}\right)$ or $B X^{\prime}(t)$ as a non-linear term with a deviating argument like $G\left(X^{\prime}(t-\tau(t))\right)$. To the best of our knowledge, it is not easy to discuss the topic for Eq. (1.2). The possible reason is that the construction or definition of a suitable Lyapunov function or functional for higher differential systems remains as an open problem in the literature by this time. This case is more difficult for the functional differential systems of higher order with variable delay. The choice of the second term in Eq.(1.2) as $G\left(X^{\prime}(t-\tau(t))\right)$ is important and the discussion of the problems for this case is some hard. This means that, in view of the whole mentioned discussion, it is worth to discuss the globally asymptotic stability / uniformly stability, boundedness and uniformly boundedness to Eq. (1.2).

Besides, this paper may be useful for researchers working on the qualitative behaviors of solutions of third differential equations and completes that in the literature. These cases show the novelty and originality of the present paper.

Consider general delay differential system

$$
\dot{x}=f\left(x_{t}\right), x_{t}=x(t+\theta),-r \leq \theta<0, t \geq 0 .
$$

Let $C=C\left([-r, 0], \mathcal{R}^{n}\right)$ denote the space of continuous function from $[-r, 0]$ into $\mathcal{R}^{n}$ and assume that $f: C \rightarrow \mathcal{R}^{n}$ is continuous function. We say that $V: C \rightarrow \mathcal{R}$ is a Lyapunov function on a set $G \subset C$ relative to $f$ if 
$V$ is continuous on $\bar{G}$, the closure of $G, \dot{V}$ is defined on $G$ and $\dot{V} \leq 0$ on $G$.

Lemma 1(Hale [62]). Suppose $f(0)=0$. Let $V$ be $a$ continuous functional defined on $C_{H}=C$ with $V(0)=0$ and let $u(s)$ be a function, non-negative and continuous for $0 \leq s<\infty, u(s) \rightarrow \infty$ as $s \rightarrow \infty$ with $u(0)=0$. If for all $\varphi \in C, u(|\phi(0)|) \leq V(\phi), \dot{V}(\varphi) \leq 0$, then the zero solution of $\dot{x}=f\left(x_{t}\right)$ is stable.

If we define $Z=\left\{\varphi \in C_{H}: \dot{V}(\varphi)=0\right\}$, then the zero solution of $\dot{x}=f\left(x_{t}\right)$ is asymptotically stable, provided that the largest invariant set in $Z$ is $\underline{\mathrm{O}}=0$.

Besides, we consider the general non-autonomous delay differential system

$$
\dot{x}=g\left(t, x_{t}\right), x_{t}=x(t+\theta),-r \leq \theta<0, t \geq 0,
$$

where $g:[0, \infty) \times C_{\rho} \rightarrow \mathcal{R}^{n}$ is a continuous mapping, $g(t, 0)=0$, and we suppose that $G$ takes closed bounded sets into bounded sets of $\mathcal{R}^{n}$. Here $(C,\|\|$.$) is the Banach space of continuous function \phi:[-r, 0] \rightarrow \mathcal{R}^{n}$ and for $\phi \in C,\|\phi\|=$ sup $_{-r \leq \theta \leq 0}|\phi(\theta)|, r>0, C_{\rho}$ is the open ball in $C$ of radius $\rho ; C_{\rho}:=\left\{\varphi \in C\left([-r, 0], \mathcal{R}^{n}\right):\|\varphi\|<\rho\right\}$.

Theorem A(Yoshizawa [63 pp.191]). Assume that there is a Lyapunov functional $V_{0}(t, x)$ for $\dot{x}=g\left(t, x_{t}\right)$, and wedges satisfying;

wedges),

(i) $W_{1}(|\phi(0)|) \leq V_{0}(t, \phi) \leq W_{2}(\|\phi\|)$, (where $W_{1}(r)$ and $W_{2}(r)$ are (ii) $\dot{V}_{0}(t, \phi) \leq 0$.

Then the zero solution of $\dot{x}=g\left(t, x_{t}\right)$ is uniformly stable.

Let $S$ be the set of $\phi \in C$ such that $\|\varphi\|>\rho$, denote by $S^{*}$ the set of all functions $\phi \in C$ such that $|\phi(0)| \geq \rho$, where $\rho$ may be large (Yoshizawa [63, pp.202]).

Theorem B (Yoshizawa [63, pp.202]). Suppose that there exists a continuous Lyapunov functional $V_{0}(t, \phi)$ defined for all $t \in \mathcal{R}^{+}$and $\phi \in S^{*}$ which 
satisfies the following conditions;

(i) $\mathrm{a}(|\phi(0)|) \leq V_{0}(t, \phi) \leq b_{1}(|\phi(0)|)+b_{2}(\|\phi\|)$, where $a(r), b_{1}(r), b_{2}(r) \in C I,(C I$ denotes the families of continuous increasing functions), and are positive for $r>H$ and $a(r)-b_{2}(r) \rightarrow \infty$ as $r \rightarrow \infty$,

(ii) $\dot{V}_{0}(t, \phi) \leq 0$. Then the solutions of $\dot{x}=g\left(t, x_{t}\right)$ are uniformly bounded.

Lemma 2. (Bellman [64, pp.288]). Let $M$ be a real symmetric $n \times n$ -matrix. Then for any $X \in \mathcal{R}^{n}$

$$
\delta_{M}\|X\|^{2} \leq\langle M X, X\rangle \leq \Delta_{M}\|X\|^{2},
$$

where $\delta_{M}$ and $\Delta_{M}$ are, respectively, positive and simple, the least and greatest eigenvalues of the matrix $M$.

\section{Stability}

We introduce some basic assumptions needed in the proofs.

(A1) $A$ is an $n \times n$ - symmetric constant matrix,

$$
\delta_{a} \leq \lambda_{i}(A) \leq \triangle_{a}
$$

where $\delta_{a}$ and $\triangle_{a}$ are positive constants.

(A2) $H(0)=0, J_{H}$ exists and is an $n \times n$-symmetric matrix,

$$
\begin{gathered}
H\left(X_{1}\right) \neq 0, \text { when } X_{1} \neq 0, \\
\delta_{h} \leq \lambda_{i}\left(J_{H}\left(X_{1}\right)\right) \leq \triangle_{h} \text { for } X_{1} \in \mathcal{R}^{n},
\end{gathered}
$$

where $\delta_{h}$ and $\triangle_{h}$ are positive constants.

(A3) $G(0)=0, J_{G}$ exists and is an $n \times n$ - symmetric matrix,

$$
\delta_{b} \leq \lambda_{i}\left(J_{G}\left(X_{2}\right)\right) \leq \triangle_{b} \text { for } X_{2} \in \mathcal{R}^{n},
$$

where $\delta_{b}$ and $\triangle_{b}$ are positive constants, and the matrices $J_{G}$ and $J_{H}$ commute with each other. 
Let $F(.) \equiv 0$. The stability result of this paper is given by the following theorem.

Theorem 1. We assume that assumptions $(A 1)-(A 3)$ hold. If

$\tau_{0}<\min \left\{\frac{2\left(\delta_{b}-\beta \triangle_{h}\right)\left(1-\tau_{1}\right)}{\left(\triangle_{b}+\triangle_{h}\right)\left(1-\tau_{1}\right)+(\beta+1) \triangle_{b}}, \frac{2\left(\beta \delta_{b}-1\right)\left(1-\tau_{1}\right)}{\beta\left(\triangle_{b}+\triangle_{h}\right)\left(1-\tau_{1}\right)+(\beta+1) \triangle_{h}}\right\}$

then the zero solution of Eq. (1.2) is globally asymptotic stable, where $\tau_{1}$ and $\beta$ are positive constants with $0<\tau_{1}<1$.

Proof. Define a functional $W_{0}=W_{0}(t)=W_{0}\left(X_{1}(t), X_{2}(t), X_{3}(t)\right)$ by

$$
\begin{gathered}
W_{0}=2 \int_{0}^{1}\left\langle H\left(\sigma X_{1}\right), X_{1}\right\rangle d \sigma+\left\langle A X_{2}, X_{2}\right\rangle+2 \beta \int_{0}^{1}\left\langle G\left(\sigma X_{2}\right), X_{2}\right\rangle d \sigma \\
+\beta\left\langle X_{3}, X_{3}\right\rangle+2\left\langle X_{2}, X_{3}\right\rangle+2 \beta\left\langle X_{2}, H\left(X_{1}\right)\right\rangle \\
+2 \lambda \int_{-\tau(t)}^{0} \int_{t+s}^{t}\left\|X_{2}(\theta)\right\|^{2} d \theta d s+2 \eta \int_{-\tau(t)}^{0} \int_{t+s}^{t}\left\|X_{3}(\theta)\right\|^{2} d \theta d s,
\end{gathered}
$$

where $\beta, \lambda$ and $\eta$ are positive constants, the constants $\lambda$ and $\eta$ will be determined later in the proof.

From assumption $\left(A_{3}\right)$, Lemma 2 and

$$
G(0)=0, \frac{\partial}{\partial \sigma} G\left(\sigma X_{2}\right)=J_{G}\left(\sigma X_{2}\right) X_{2}
$$

We obtain

$2 \int_{0}^{1}\left\langle G\left(\sigma X_{2}\right), X_{2}\right\rangle d \sigma=2 \int_{0}^{1} \int_{0}^{1} \sigma_{1}\left\langle J_{G}\left(\sigma_{1} \sigma_{2} X_{2}\right) X_{2}, X_{2}\right\rangle d \sigma_{1} d \sigma_{2} \geq \delta_{b}\left\|X_{2}\right\|^{2}$.

Similarly, it follows from

$$
H(0)=0, \frac{\partial}{\partial \sigma_{1}} H\left(\sigma_{1} X_{1}\right)=J_{H}\left(\sigma_{1} X_{1}\right) X_{1}
$$

that

$$
\frac{\partial}{\partial \sigma_{1}}\left\langle H\left(\sigma_{1} X_{1}\right), H\left(\sigma_{1} X_{1}\right)\right\rangle=2\left\langle J_{H}\left(\sigma_{1} X_{1}\right) X_{1}, H\left(\sigma_{1} X_{1}\right)\right\rangle .
$$


Integrating, from $\sigma_{1}=0$ to $\sigma_{1}=1$, the both sides of the last estimates, respectively, we get

$$
H\left(X_{1}\right)=\int_{0}^{1} J_{H}\left(\sigma_{1} X_{1}\right) X_{1} d \sigma_{1}
$$

and

$$
\left\langle H\left(X_{1}\right), H\left(X_{1}\right)\right\rangle=2 \int_{0}^{1}\left\langle J_{H}\left(\sigma_{1} X_{1}\right) X_{1}, H\left(\sigma_{1} X_{1}\right)\right\rangle d \sigma_{1}
$$

It can also be seen that

$$
2 \int_{0}^{1}\left\langle H\left(\sigma_{1} X_{1}\right), X_{1}\right\rangle d \sigma_{1}=2 \int_{0}^{1} \int_{0}^{1} \sigma_{1}\left\langle J_{H}\left(\sigma_{1} \sigma_{2} X_{1}\right) X_{1}, X_{1}\right\rangle d \sigma_{1} d \sigma_{2} .
$$

Further, it is obvious that

$$
\frac{\partial}{\partial \sigma_{2}}\left\langle H\left(\sigma_{1} \sigma_{2} X_{1}\right), J_{H}\left(\sigma_{1} X_{1}\right) X_{1}\right\rangle=\left\langle\sigma_{1} J_{H}\left(\sigma_{1} X_{1}\right) X_{1}, J_{H}\left(\sigma_{1} X_{1}\right) X_{1}\right\rangle .
$$

Integrating the both sides of the last equality from $\sigma_{2}=0$ to $\sigma_{2}=1$, we obtain

$$
\left\langle H\left(\sigma_{1} X_{1}\right), J_{H}\left(\sigma_{1} X_{1}\right) X_{1}\right\rangle=\int_{0}^{1}\left\langle\sigma_{1} J_{H}\left(\sigma_{1} X_{1}\right) X_{1}, J_{H}\left(\sigma_{1} X_{1}\right) X_{1}\right\rangle d \sigma_{2} .
$$

Then, we have

$$
\left\langle H\left(X_{1}\right), H\left(X_{1}\right)\right\rangle=2 \int_{0}^{1} \int_{0}^{1}\left\langle\sigma_{1} J_{H}\left(\sigma_{1} X_{1}\right) X_{1}, J_{H}\left(\sigma_{1} X_{1}\right) X_{1}\right\rangle d \sigma_{1} d \sigma_{2}
$$

From (2.1), the above discussion, Lemma 2 and the assumptions of Theorem 1, we can obtain 


$$
\begin{aligned}
& 2 W_{0} \geq 2 \int_{0}^{1}\left\langle H\left(\sigma X_{1}\right), X_{1}\right\rangle d \sigma+\left\langle A X_{2}, X_{2}\right\rangle+\beta \delta_{b}\left\|X_{2}\right\|^{2} \\
& +\beta\left\langle X_{3}, X_{3}\right\rangle+2\left\langle X_{2}, X_{3}\right\rangle+2 \beta\left\langle X_{2}, H\left(X_{1}\right)\right\rangle \\
& =2 \int_{0}^{1}\left\langle H\left(\sigma X_{1}\right), X_{1}\right\rangle d \sigma-\beta \delta_{b}^{-1}\left\langle H\left(X_{1}\right), H\left(X_{1}\right)\right\rangle \\
& +\beta\left\|\delta_{b}^{\frac{1}{2}} X_{2}+\delta_{b}^{-\frac{1}{2}} H\left(X_{1}\right)\right\|^{2}+\beta\left\|X_{3}+\beta^{-1} X_{2}\right\|^{2} \\
& +\left\langle\left(A-\beta^{-1} I\right) X_{2}, X_{2}\right\rangle \\
& \geq 2 \int_{0}^{1}\left\langle H\left(\sigma X_{1}\right), X_{1}\right\rangle d \sigma-\beta \delta_{b}^{-1}\left\langle H\left(X_{1}\right), H\left(X_{1}\right)\right\rangle \\
& +\beta\left\|X_{3}+\beta^{-1} X_{2}\right\|^{2}+\left\langle\left(A-\beta^{-1} I\right) X_{2}, X_{2}\right\rangle \\
& \geq 2 \int_{0}^{1} \int_{0}^{1} \sigma_{1}\left\langle J_{H}\left(\sigma_{1} \sigma_{2} X_{1}\right) X_{1}, X_{1}\right\rangle d \sigma_{1} d \sigma_{2}+\beta\left\|X_{3}+\beta^{-1} X_{2}\right\|^{2} \\
& -2 \beta \delta_{b}^{-1} \int_{0}^{1} \int_{0}^{1}\left\langle\sigma_{1} J_{H}\left(\sigma_{1} X_{1}\right) X_{1}, J_{H}\left(\sigma_{1} X_{1}\right) X_{1}\right\rangle d \sigma_{1} d \sigma_{2} \\
& +\left\langle\left(A-\beta^{-1} I\right) X_{2}, X_{2}\right\rangle \\
& \left.\geq\left(1-\beta \delta_{b}^{-1} \Delta_{h}\right) \delta_{h}\right)\left\|X_{1}\right\|^{2}+\beta\left\|X_{3}+\beta^{-1} X_{2}\right\|^{2}+\left(\delta_{a}-\beta^{-1}\right)\left\|X_{2}\right\|^{2} .
\end{aligned}
$$

It is clear all the coefficients in the last inequality are positive and hence there exists a positive constant $K$ such that

$$
W_{0} \geq K\left(\left\|X_{1}\right\|^{2}+\left\|X_{2}\right\|^{2}+\left\|X_{3}\right\|^{2}\right)
$$

and

$$
\left\|X_{1}\right\|^{2}+\left\|X_{2}\right\|^{2}+\left\|X_{3}\right\|^{2} \leq K^{-1} W_{0} .
$$

For the time derivative the functional $W_{0}$ by a straightforward calculation from (1.3) and (2.1), we obtain 


$$
\begin{aligned}
& \dot{W}_{0}(t)=-\left\langle(\beta A-I) X_{3}, X_{3}\right\rangle-\left\langle G\left(X_{2}\right), X_{2}\right\rangle+\beta\left\langle J_{H}\left(X_{1}\right) X_{2}, X_{2}\right\rangle \\
& +\beta \int_{t-\tau(t)}^{t}\left\langle X_{3}(t), J_{G}\left(X_{2}(s)\right) X_{3}(s)\right\rangle d s+\int_{t-\tau(t)}^{t}\left\langle X_{2}(s), J_{G}\left(X_{2}(s)\right) X_{3}(s)\right\rangle d s \\
& +\beta \int_{t-\tau(t)}^{t}\left\langle X_{3}(t), J_{H}\left(X_{1}(s)\right) X_{2}(s)\right\rangle d s+\int_{t-\tau(t)}^{t}\left\langle X_{2}(t), J_{H}\left(X_{1}(s)\right) X_{2}(s)\right\rangle d s \\
& +\lambda \tau(t)\left\|X_{2}\right\|^{2}+\eta \tau(t)\left\|X_{3}\right\|^{2}-\lambda\left(1-\tau^{\prime}(t)\right) \int_{t-\tau(t)}^{t}\left\|X_{2}(\theta)\right\|^{2} d \theta \\
& -\eta\left(1-\tau^{\prime}(t)\right) \int_{t-\tau(t)}^{t}\left\|X_{3}(\theta)\right\|^{2} d \theta
\end{aligned}
$$

The assumptions of Theorem $1,0 \leq \tau(t) \leq \tau_{0}$ and $\tau^{\prime}(t) \leq \tau_{1}$, $0<\tau_{1}<1$, imply that

$$
\begin{gathered}
\left\langle(\beta A-I) X_{3}, X_{3}\right\rangle \geq\left(\beta \delta_{a}-1\right)\left\|X_{3}\right\|^{2}, \\
\left\langle G\left(X_{2}\right), X_{2}\right\rangle=\int_{0}^{1}\left\langle J_{G}\left(\sigma X_{2}\right) X_{2}, X_{2}\right\rangle d \sigma \\
\geq \int_{0}^{1}\left\langle\delta_{b} X_{2}, X_{2}\right\rangle d \sigma=\delta_{b}\left\|X_{2}\right\|^{2}, \\
\beta\left\langle J_{H}\left(X_{1}\right) X_{2}, X_{2}\right\rangle \leq \beta \Delta_{h}\left\|X_{2}\right\|^{2}, \\
\beta \int_{t-\tau(t)}^{t}\left\langle X_{3}(t), J_{G}\left(X_{2}(s)\right) X_{3}(s)\right\rangle d s \leq \beta\left\|X_{3}(t)\right\| \int_{t-\tau(t)}^{t}\left\|J_{G}\left(X_{2}(s)\right)\right\|\left\|X_{3}(s)\right\| d s \\
\leq \beta \Delta_{b}\left\|X_{3}(t)\right\| \int_{t-\tau(t)}^{t}\left\|X_{3}(s)\right\| d s \\
\leq \frac{1}{2} \beta \Delta_{b} \int_{t-\tau(t)}^{t}\left\{\left\|X_{3}(t)\right\|^{2}+\left\|X_{3}(s)\right\|^{2}\right\} d s \\
=\frac{1}{2} \beta \Delta_{b} \tau(t)\left\|X_{3}\right\|^{2}+\frac{1}{2} \beta \Delta_{b} \int_{t-\tau(t)}^{t}\left\|X_{3}(s)\right\|^{2} d s \\
\leq \frac{1}{2} \beta \Delta_{b} \tau_{0}\left\|X_{3}\right\|^{2}+\frac{1}{2} \beta \Delta_{b} \int_{t-\tau(t)}^{t}\left\|X_{3}(s)\right\|^{2} d s,
\end{gathered}
$$




$$
\begin{aligned}
& \int_{t-\tau(t)}^{t}\left\langle X_{2}(t), J_{G}\left(X_{2}(s)\right) X_{3}(s)\right\rangle d s \leq\left\|X_{2}(t)\right\| \int_{t-\tau(t)}^{t}\left\|J_{G}\left(X_{2}(s)\right)\right\|\left\|X_{3}(s)\right\| d s \\
& \leq \Delta_{b}\left\|X_{2}(t)\right\| \int_{t-\tau(t)}^{t}\left\|X_{3}(s)\right\| d s \\
& \leq \frac{1}{2} \Delta_{b} \int_{t-\tau(t)}^{t}\left\{\left\|X_{2}(t)\right\|^{2}+\left\|X_{3}(s)\right\|^{2}\right\} d s \\
& =\frac{1}{2} \Delta_{b} \tau(t)\left\|X_{2}\right\|^{2}+\frac{1}{2} \Delta_{b} \int_{t-\tau(t)}^{t}\left\|X_{3}(s)\right\|^{2} d s \\
& \leq \frac{1}{2} \Delta_{b} \tau_{0}\left\|X_{2}\right\|^{2}+\frac{1}{2} \Delta_{b} \int_{t-\tau(t)}^{t}\left\|X_{3}(s)\right\|^{2} d s, \\
& \beta \int_{t-\tau(t)}^{t}\left\langle X_{3}(t), J_{H}\left(X_{1}(s)\right) X_{2}(s)\right\rangle d s \leq \beta\left\|X_{3}(t)\right\| \int_{t-\tau(t)}^{t}\left\|J_{H}\left(X_{1}(s)\right)\right\|\left\|X_{2}(s)\right\| d s \\
& \leq \beta \Delta_{h}\left\|X_{3}(t)\right\| \int_{t-\tau(t)}^{t}\left\|X_{2}(s)\right\| d s \\
& \leq \frac{1}{2} \beta \Delta_{h} \int_{t-\tau(t)}^{t}\left\{\left\|X_{3}(t)\right\|^{2}+\left\|X_{2}(s)\right\|^{2}\right\} d s \\
& =\frac{1}{2} \beta \Delta_{h} \tau_{0}\left\|X_{3}\right\|^{2}+\frac{1}{2} \beta_{h} \int_{t-\tau(t)}^{t}\left\|X_{2}(s)\right\|^{2} d s, \\
& \int_{t-\tau(t)}^{t}\left\langle X_{2}(t), J_{H}\left(X_{1}(s)\right) X_{2}(s)\right\rangle d s \leq\left\|X_{2}(t)\right\| \int_{t-\tau(t)}^{t}\left\|J_{H}\left(X_{1}(s)\right)\right\|\left\|X_{2}(s)\right\| d s \\
& \leq \Delta_{h}\left\|X_{2}(t)\right\| \int_{t-\tau(t)}^{t}\left\|X_{2}(s)\right\| d s \\
& \leq \frac{1}{2} \Delta_{h} \int_{t-\tau(t)}^{t}\left\{\left\|X_{2}(t)\right\|^{2}+\left\|X_{2}(s)\right\|^{2}\right\} d s \\
& =\frac{1}{2} \Delta_{h} \tau(t)\left\|X_{2}\right\|^{2}+\frac{1}{2} \Delta_{h} \int_{t-\tau(t)}^{t}\left\|X_{2}(s)\right\|^{2} d s \\
& \leq \frac{1}{2} \Delta_{h} \tau_{0}\left\|X_{2}\right\|^{2}+\frac{1}{2} \Delta_{h} \int_{t-\tau(t)}^{t}\left\|X_{2}(s)\right\|^{2} d s, \\
& \lambda \tau(t)\left\|X_{2}\right\|^{2} \leq \lambda \tau_{0}\left\|X_{2}\right\|^{2}, \\
& \eta \tau(t)\left\|X_{3}\right\|^{2} \leq \eta \tau_{0}\left\|X_{3}\right\|^{2} \\
& -\lambda\left(1-\tau^{\prime}(t)\right) \int_{t-\tau(t)}^{t}\left\|X_{2}(\theta)\right\|^{2} d \theta \leq-\lambda\left(1-\tau_{1}\right) \int_{t-\tau(t)}^{t}\left\|X_{2}(\theta)\right\|^{2} d \theta, \\
& -\eta\left(1-\tau^{\prime}(t)\right) \int_{t-\tau(t)}^{t}\left\|X_{3}(\theta)\right\|^{2} d \theta \leq-\eta\left(1-\tau_{1}\right) \int_{t-\tau(t)}^{t}\left\|X_{3}(\theta)\right\|^{2} d \theta .
\end{aligned}
$$


On gathering the obtained inequalities into $\dot{W}_{0}(t)$, we arrive at

$$
\begin{gathered}
\dot{W}_{0}(t) \leq-\left\{\delta_{b}-\beta \Delta_{h}-\frac{1}{2}\left(\Delta_{b}+\Delta_{h}+2 \lambda\right) \tau_{0}\right\}\left\|X_{2}\right\|^{2} \\
-\left\{\left(\beta \delta_{a}-1\right)-\frac{1}{2}\left(\beta\left(\Delta_{b}+\Delta_{h}\right)+2 \eta\right) \tau_{0}\right\}\left\|X_{3}\right\|^{2} \\
+\left\{\frac{1}{2}(\beta+1) \Delta_{h}-\lambda\left(1-\tau_{1}\right)\right\} \int_{t-\tau(t)}^{t}\left\|X_{2}(\theta)\right\|^{2} d s \\
+\left\{\frac{1}{2}(\beta+1) \Delta_{b}-\eta\left(1-\tau_{1}\right)\right\} \int_{t-\tau(t)}^{t}\left\|X_{3}(\theta)\right\|^{2} d s .
\end{gathered}
$$

Let

$$
\lambda=\frac{(\beta+1) \Delta_{h}}{2\left(1-\tau_{1}\right)}
$$

and

$$
\eta=\frac{(\beta+1) \Delta_{b}}{2\left(1-\tau_{1}\right)}
$$

Hence

If

$$
\begin{gathered}
\dot{W}_{0}(t) \leq-\left\{\delta_{b}-\beta \Delta_{h}-\frac{1}{2}\left(\frac{\left(\Delta_{b}+\Delta_{h}\right)\left(1-\tau_{1}\right)+(\beta+1) \Delta_{h}}{1-\tau_{1}}\right) \tau_{0}\right\}\left\|X_{2}\right\|^{2} \\
-\left\{\left(\beta \delta_{b}-1\right)-\frac{1}{2}\left(\frac{\beta\left(\Delta_{b}+\Delta_{h}\right)\left(1-\tau_{1}\right)+(\beta+1) \Delta_{b}}{1-\tau_{1}}\right) \tau_{0}\right\}\left\|X_{3}\right\|^{2} .
\end{gathered}
$$

$\tau_{0}<\min \left\{\frac{2\left(\delta_{b}-\beta \triangle_{h}\right)\left(1-\tau_{1}\right)}{\left(\triangle_{b}+\triangle_{h}\right)\left(1-\tau_{1}\right)+(\beta+1) \triangle_{b}}, \frac{2\left(\beta \delta_{b}-1\right)\left(1-\tau_{1}\right)}{\beta\left(\triangle_{b}+\triangle_{h}\right)\left(1-\tau_{1}\right)+(\beta+1) \triangle_{h}}\right\}$,

$0<\tau_{1}<1$

then

$$
\dot{W}_{0}(t) \leq-K_{1}\left\|X_{2}\right\|^{2}-K_{2}\left\|X_{3}\right\|^{2} \leq 0
$$

for some positive constants $K_{1}$ and $K_{2}$. In addition, we can easily see that

$$
W_{0} \quad\left(X_{1}, X_{2}, X_{3}\right) \rightarrow \infty \quad \text { as } \quad\left\|X_{1}\right\|^{2}+\left\|X_{2}\right\|^{2}+\left\|X_{3}\right\|^{2} \rightarrow \infty .
$$

Consider the set defined by

$$
Q \equiv\left\{\left(X_{1}, X_{2}, X_{3}\right): \dot{W}_{0}\left(X_{1}, X_{2}, X_{3}\right)=0\right\} .
$$


When we apply the LaSalle's invariance principle, we observe that $\left(X_{1}, X_{2}, X_{3}\right) \in Q$ implies that $X_{2}=X_{3}=0$ and hence $X_{1}=\mu,(\mu \neq 0$ is a constant vector). From the last estimate and system (1.3), we have $H(\mu)=0$, which necessarily implies that $\mu=0$ since $H(0)=0$. Therefore

$$
X_{1}=X_{2}=X_{3}=0 \text {. }
$$

In fact, this result implies that the largest invariant set contained in $Q$ is $(0,0,0) \in Q$. By Lemma 1 , we conclude that the zero solution of system (1.3) is asymptotically stable. Hence, the zero solution of Eq. (1.2) is the globally asymptotic stable. This completes the proof of Theorem 1.

Theorem 2. If assumptions $(A 1)-(A 3)$ and

$\tau_{0}<\min \left\{\frac{2\left(\delta_{b}-\beta \triangle_{h}\right)\left(1-\tau_{1}\right)}{\left(\triangle_{b}+\triangle_{h}\right)\left(1-\tau_{1}\right)+(\beta+1) \triangle_{b}}, \frac{2\left(\beta \delta_{b}-1\right)\left(1-\tau_{1}\right)}{\beta\left(\triangle_{b}+\triangle_{h}\right)\left(1-\tau_{1}\right)+(\beta+1) \triangle_{h}}\right\}$,

hold, then the zero solution of Eq. (1.2) is uniformly stable, where $\tau_{1}$ and $\beta$ are positive constant with $0<\tau_{1}<1$.

Proof. To prove Theorem 2, our main tool is the functional $W_{0}$ given by (2.1). It is clear from the proof of Theorem 1 that the functional $W_{0}$ and its time derivative satisfy the assumptions of Theorem A, except $W_{2}(\|\phi\|)$;

$$
W_{1}(|\phi(0)|) \leq W_{0} \leq W_{2}(\|\phi\|), \dot{W}(\phi) \leq 0 .
$$

Besides, subject to the assumptions of Theorem 2, it can be easily obtained that $W_{0} \leq W_{2}(\|\phi\|)$ We omit the detail of the proof. This completes the proof of Theorem 2 .

\section{Boundedness}

Let $F() \neq$.0 . The boundedness results of this paper are given by the following theorems.

Theorem 3. We assume that all the assumptions of Theorem 1 hold, except $F(.) \equiv 0$. Further, we suppose that there exists a non-negative and continuous function $P=P(t)$ such that

$\left\|F\left(t, X_{1}, X_{2}, X_{3}\right)\right\| \leq P(t)$ for all $t \geq 0, \max P(t)<\infty$ and $P \in L^{1}(0, \infty)$, 
where $L^{1}(0, \infty)$ denotes the space of Lebesgue integrable functions.

If

$\tau_{0}<\min \left\{\frac{2\left(\delta_{b}-\beta \triangle_{h}\right)\left(1-\tau_{1}\right)}{\left(\triangle_{b}+\triangle_{h}\right)\left(1-\tau_{1}\right)+(\beta+1) \triangle_{b}}, \frac{2\left(\beta \delta_{b}-1\right)\left(1-\tau_{1}\right)}{\beta\left(\triangle_{b}+\triangle_{h}\right)\left(1-\tau_{1}\right)+(\beta+1) \triangle_{h}}\right\}$,

$0<\tau_{1}<1$

then there exists a constant $M>0$ such that any solution $\left(X_{1}(t), X_{2}(t), X_{3}(t)\right)$ of system (1.3) determined by

$$
X_{1}(0)=X_{10}, X_{2}(0)=X_{20}, X_{3}(0)=X_{30}
$$

satisfies

$$
\left\|X_{1}(t)\right\| \leq M,\left\|X_{2}(t)\right\| \leq M,\left\|X_{3}(t)\right\| \leq M
$$

for all $t \in \mathcal{R}^{+}$.

Proof. Let $F()=.F\left(t, X_{1}, X_{2}, X_{3}\right)$. For the case of $F() \neq$.0 , it can be concluded that

$$
\dot{W}_{0}(t) \leq-K_{1}\left\|X_{2}\right\|^{2}-K_{2}\left\|X_{3}\right\|^{2}+\left\langle X_{2}, F(.)\right\rangle+\left\langle\beta X_{3}, F(.)\right\rangle .
$$

Then

$$
\begin{aligned}
& \dot{W}_{0}(t) \leq\left(\left\|X_{2}\right\|+\beta\left\|X_{3}\right\|\right)\|F(.)\| \\
& \quad \leq K_{3}\left(\left\|X_{2}\right\|+\left\|X_{3}\right\|\right)\|F(.)\| \\
& \quad \leq K_{3}\left(2+\left\|X_{2}\right\|^{2}+\left\|X_{3}\right\|^{2}\right) P(t) \\
& \quad \leq 2 K_{3} P(t)+K^{-1} K_{3} W_{0}(t) P(t),
\end{aligned}
$$

by the assumptions of Theorem 3 and (2.2), where

$$
K_{3}=\max \{1, \beta\} .
$$

The integration of both sides of the last inequality, between 0 to $t$, $(t \geq 0)$, leads that

$$
W_{0}(t) \leq W_{0}(0)+2 K_{3} \int_{0}^{t} P(s) d s+K^{-1} K_{3} \int_{0}^{t} W_{0}(s) P(s) d s .
$$

Let

$$
M=W_{0}(0)+2 K_{3} \int_{0}^{\infty} P(s) d s .
$$


Then

$$
W_{0}(t) \leq M+K^{-1} K_{3} \int_{0}^{\infty} W_{0}(s) P(s) d s .
$$

By noting the Gronwall-Bellman inequality (see Ahmad and Rama Mohana Rao [9, pp.41]), we can get

$$
W_{0}(t) \leq M \exp \left(K^{-1} K_{3} \int_{0}^{\infty} P(s) d s\right) .
$$

By the estimate $\left\|X_{1}\right\|^{2}+\left\|X_{2}\right\|^{2}+\left\|X_{3}\right\|^{2} \leq K^{-1} W_{0}$ and the assumption $P \in L^{1}(0, \infty)$, we can conclude that all solutions of system (1.3) are bounded. This completes the proof of Theorem 3 .

Theorem 4. If the assumptions Theorem 3 and

$\tau_{0}<\min \left\{\frac{2\left(\delta_{b}-\beta \triangle_{h}\right)\left(1-\tau_{1}\right)}{\left(\triangle_{b}+\triangle_{h}\right)\left(1-\tau_{1}\right)+(\beta+1) \triangle_{b}}, \frac{2\left(\beta \delta_{b}-1\right)\left(1-\tau_{1}\right)}{\beta\left(\triangle_{b}+\triangle_{h}\right)\left(1-\tau_{1}\right)+(\beta+1) \triangle_{h}}\right\}$

hold, then the zero solution of Eq. (1.2) is uniformly bounded, where $\tau_{1}$ is positive constant with $0<\tau_{1}<1$.

Proof. To complete the poof of Theorem 4, the main tool is the functional $W_{0}$ given by $(2.1)$. When we benefit from the functional $W_{0}$ and the assumptions of Theorem 4, we can easily complete the poof of Theorem 4 . Therefore, we omit the details of the proof.

\section{Conclusion}

We consider a functional differential system of third order with variable delay. We investigate the globally asymptotic stability/uniformly stability/boundedness/ uniformly boundedness of solutions. The technique of proofs involves defining an appropriate Lyapunov functional. Our results include, improve and complete some recent results in the literature. 


\section{References}

[1] R. Reissig, G. Sansone, R. Conti, Non-linear differential equations of higher order. Translated from the German. Noordhoff International Publishing, Leyden, 1974.

[2] A. T. Ademola, P. O. Arawomo, Stability and ultimate boundedness of solutions to certain third-order differential equations. Appl. Math. E-Notes 10, pp. 61-69, (2010).

[3] A. T. Ademola, P. O. Arawomo, Stability and uniform ultimate boundedness of solutions of some third-order differential equations. Acta Math. Acad. Paedagog. Nyhzi. (N.S.) 27, No. 1, pp. 51-59, (2011).

[4] A. T. Ademola, P. O. Arawomo, Asymptotic behaviour of solutions of third order nonlinear differential equations. Acta Univ. Sapientiae Math. 3, No. 2, pp. 197-211, (2011).

[5] A. T. Ademola, P. O. Arawomo, Uniform stability and boundedness of solutions of nonlinear delay differential equations of the third order. Math. J. Okayama Univ. 55, pp. 157-166, (2013).

[6] A. T. Ademola, B. S. Ogundare, M. O. Ogundiran, O. A. Adesina, Stability, boundedness, and existence of periodic solutions to certain third-order delay differential equations with multiple deviating arguments. Int. J. Differ. Equ., Art. ID 213935, 12 pp...., (2015).

[7] A. U. Afuwape, J. E. Castellanos, Asymptotic and exponential stability of certain third- order non-linear delayed differential equations: frequency domain method. Appl. Math. Comput. 216, No. 3, pp. 940950, (2010).

[8] A. U. Afuwape, M. O. Omeike, Stability and boundedness of solutions of a kind of third- order delay differential equations. Comput. Appl. Math. 29, No. 3, pp. 329-342, (2010).

[9] S. Ahmad, M. Rama Mohana Rao, Theory of ordinary differential equations. With applications in biology and engineering. Affiliated East-West Press Pvt. Ltd., New Delhi, (1999). 
[10] Y. Bai, C. Guo, New results on stability and boundedness of third order nonlinear delay differential equations. Dynam. Systems Appl. 22, No. 1, pp. 95-104, (2013).

[11] J. O. C. Ezeilo, On the stability of solutions of certain differential equations of the third order. Quart. J. Math. Oxford Ser. (2) 11, pp. 64-69, (1960).

[12] J. O. C. Ezeilo, H. O. Tjumla, Further results for a system of third order differential equations. Atti Accad. Naz. Lincei Rend. Cl. Sci. Fis. Mat. Natur. (8) 58, No. 2, pp. 143-151, (1975).

[13] J. R. Graef, D. Beldjerd, M. Remili, On stability, ultimate boundedness, and existence of periodic solutions of certain third order differential equations with delay. PanAmer. Math. J. 25, No. 1, pp. 82-94, (2015).

[14] J. R. Graef, L. D. Oudjedi, M. Remili, Stability and square integrability of solutions of nonlinear third order differential equations. Dyn. Contin. Discrete Impuls. Syst. Ser. A Math. Anal. 22, No. 4, pp. 313324, (2015).

[15] ] J. R. Graef, C. Tunc, Global asymptotic stability and boundedness of certain multidelay functional differential equations of third order. Math. Methods Appl. Sci. 38, No. 17, pp. 3747-3752, (2015).

[16] E. Korkmaz, C. Tunc, Convergence to non-autonomous differential equations of second order. J. Egyptian Math. Soc. 23, No. 1, pp. 2730, (2015).

[17] A. M. Mahmoud, C. Tunc, Stability and boundedness of solutions of a certain n- dimensional nonlinear delay differential system of thirdorder. Adv. Pure Appl. Math. 7(1), pp. 1-11, (2016).

[18] B. S. Ogundare, On boundedness and stability of solutions of certain third order delay differential equation. J. Nigerian Math. Soc. 31, pp. 55-68, (2012).

[19] B. S. Ogundare, J. A. Ayanjinmi, O. A. Adesina, Bounded and L 2solutions of certain third order non-linear differential equation with a square integrable forcing term. Kragujevac J. Math. 29, pp. 151-156, (2006). 
[20] A. L. Olutimo, Stability and ultimate boundedness of solutions of a certain third order nonlinear vector differential equation. J. Nigerian Math. Soc. 31, pp. 69-80, (2012).

[21] M. O. Omeike, Stability and boundedness of solutions of a certain system of third order nonlinear delay differential equations. Acta Univ. Palack. Olomuc. Fac. Rerum Natur. Math. 54, No. 1, pp. 109-119, (2015).

[22] C. Qian, On global stability of third-order nonlinear differential equations. Nonlinear Anal. 42, No. 4, Ser. A: Theory Methods, pp. 651-661, (2000).

[23] M. Remili, L. D. Oudjedi, Stability and boundedness of the solutions of non-autonomous third order differential equations with delay. Acta Univ. Palack. Olomuc. Fac. Rerum Natur. Math. 53, No. 2, pp. 139147, (2014).

[24] A. I. Sadek, Stability and boundedness of a kind of third-order delay differential system. Appl. Math. Lett. 16, No. 5, 657-662, (2003).

[25] K. E. Swick, Asymptotic behavior of the solutions of certain third order differential equations. SIAM J. Appl. Math. 19, pp. 96-102, (1970).

[26] H. O. Tejumola, B. Tchegnani, Stability, boundedness and existence of periodic solutions of some third and fourth order nonlinear delay differential equations. J. Nigerian Math. Soc. 19, pp. 9-19, (2000).

[27] C. Tunc, On the boundedness and periodicity of the solutions of a certain vector differential equation of third-order. Chinese translation in Appl. Math. Mech. 20, No. 2, pp. 153-160, (1999). Appl. Math. Mech. (English Ed.) 20, No. 2, pp. 163-170, (1999).

[28] C. Tunc, Uniform ultimate boundedness of the solutions of third-order nonlinear differential equations. Kuwait J. Sci. Engrg. 32, No. 1, pp. $39-48,(2005)$.

[29] C. Tunc, Boundedness of solutions of a third-order nonlinear differential equation. JIPAM. J. Inequal. Pure Appl. Math. 6, o. 1, Article 3, 6 pp., (2005).

[30] C. Tunc, On the asymptotic behavior of solutions of certain third-order nonlinear differential equations. J. Appl. Math. Stoch. Anal., No. 1, pp. 29-35, (2005). 
[31] C. Tunc, New results about stability and boundedness of solutions of certain non-linear third-order delay differential equations. Arab. J. Sci. Eng. Sect. A Sci. 31, No. 2, pp. 185-196, (2006).

[32] C. Tunc, On the boundedness of solutions of certain nonlinear vector differential equations of third order. Bull. Math. Soc. Sci. Math. Roumanie (N. S.) 49 (97), No. 3, pp. 291-300, (2006).

[33] C. Tunc, On the stability and boundedness of solutions to third order nonlinear differential equations with retarded argument. Nonlinear Dynam. 57, No. 1-2, pp. 97-106, (2009).

[34] C. Tunc, On the stability and boundedness of solutions of nonlinear vector differential equations of third order. Nonlinear Anal. 70, No. 6, pp. 2232-2236, (2009).

[35] C. Tunc, Bounded solutions to nonlinear delay differential equations of third order. Topol. Methods Nonlinear Anal. 34, No. 1, pp. 131-139, (2009).

[36] C. Tunc, On the stability and boundedness of solutions of nonlinear third order differential equations with delay. Filomat 24, No. 3, pp. 1-10, (2010).

[37] C. Tunc, Stability and bounded of solutions to non-autonomous delay differential equations of third order. Nonlinear Dynam. 62, No. 4, pp. 945-953, (2010).

[38] C. Tunc, On some qualitative behaviors of solutions to a kind of third order nonlinear delay differential equations. Electron. J. Qual. Theory Differ. Equ., No. 12, 19 pp. , (2010).

[39] C. Tunc, Stability and boundedness for a kind of non-autonomous differential equations with constant delay. Appl. Math. Inf. Sci. 7, No. 1, pp. 355-361, (2013).

[40] C. Tunc, Stability and boundedness of the nonlinear differential equations of third order with multiple deviating arguments. Afr. Mat. 24, No. 3, pp. 381-390, (2013).

[41] C. Tunc, On the qualitative behaviors of solutions of some differential equations of higher order with multiple deviating arguments. J. Franklin Inst. 351, No. 2, pp. 643-655, (2014). 
[42] C. Tunc, On the stability and boundedness of certain third order nonautonomous differential equations of retarded type. Proyecciones 34, No. 2, pp. 147-159, (2015).

[43] C. Tunc, Global stability and boundedness of solutions to differential equations of third order with multiple delays. Dynam. Systems Appl. 24, pp. 467-478, (2015).

[44] C. Tunc, Boundedness of solutions to certain system of differential equations with multiple delays. Mathematical Modeling and Applications in Nonlinear Dynamics. Springer Book Series, Chapter 5, pp. 109-123, (2016).

[45] C. Tunc, M. Ate, Stability and boundedness results for solutions of certain third order nonlinear vector differential equations. Nonlinear Dynam. 45, No. 3-4, pp. 273-281, (2006).

[46] C. Tunc, M. Gzen, Stability and uniform boundedness in multidelay functional differential equations of third order. Abstr. Appl. Anal., Art. ID 248717, 7 pp. - , (2013).

[47] C. Tunc, S. A. Mohammed, On the qualitative properties of differential equations of third order with retarded argument. Proyecciones 33, No. 3, pp. 325-347, (2014).

[48] C. Tunc; E. Tunc, New ultimate boundedness and periodicity results for certain third- order nonlinear vector differential equations. Math. J. Okayama Univ. 48, pp. 159-172, (2006).

[49] L. Zhang; L. Yu, Global asymptotic stability of certain third-order nonlinear differential equations. Math. Methods Appl. Sci. Math. Methods Appl. Sci. 36, No. 14, pp. 1845-1850, (2013).

[50] Y. F. Zhu, On stability, boundedness and existence of periodic solution of a kind of third order nonlinear delay differential system. Ann. Differential Equations 8, No. 2, pp. 249-259, (1992).

[51] A. U. Afuwape, P. Omari, F. Zanolin, Nonlinear perturbations of differential operators with nontrivial kernel and applications to third-order periodic boundary value problems. J. Math. Anal. Appl. 143, No. 1, pp. 35-56, (1989). 
[52] J. Andres, Periodic boundary value problem for certain nonlinear differential equations of the third order. Math. Slovaca 35, No. 3, 305-309, (1985).

[53] K. O. Fridedrichs, On nonlinear vibrations of third order. Studies in Nonlinear Vibration Theory, pp. 65-103. Institute for Mathematics and Mechanics, New York University, (1946).

[54] A. O. E. Animalu, J. O. C. Ezeilo, Some third order differential equations in physics. Fundamental open problems in science at the end of the millennium, Vol. IIII (Beijing, 1997), pp. 575-586, Hadronic Press, Palm Harbor, FL, (1999).

[55] J. O. C. Ezeilo, J. Onyia, Nonresonant oscillations for some third-order differential equations. J. Nigerian Math. Soc. 3 (1984), 83-96 (1986).

[56] K. E. Chlouverakis, J. C. Sprott, Chaotic hyperjerk systems. Chaos Solitons Fractals 28 (2006), no. 3, 739-746.

[57] R. Eichhorn, S. J. Linz, P. Hnggi, Transformations of nonlinear dynamical systems to jerky motion and its application to minimal chaotic flows. Phys Rev E 58 (1998), 7151-7164.

[58] S. J. Linz, On hyperjerky systems. Chaos Solitons Fractals 37 (2008), no. $3,741-747$.

[59] J. Cronin-Scanlon, Some mathematics of biological oscillations. SIAM Rev. 19 (1977), no. 1, 100138.

[60] L. L. Rauch, Oscillation of a third order nonlinear autonomous system. Contributions to the Theory of Nonlinear Oscillations, pp. 39-88. Annals of Mathematics Studies, no. 20. Princeton University Press, Princeton, N. J., (1950).

[61] H. Smith, An introduction to delay differential equations with applications to the life sciences. Texts in Applied Mathematics, 57. Springer, New York, (2011).

[62] J. Hale, Sufficient conditions for stability and instability of autonomous functional- differential equations. J. Differential Equations 1, pp. 452482, (1965). 
[63] T. Yoshizawa, Stability theory by Liapunov's second method. Publications of the Mathematical Society of Japan, No. 9. The Mathematical Society of Japan, Tokyo (1966).

[64] R. Bellman, Richard Introduction to matrix analysis. Reprint of the second edition 1970. With a foreword by Gene Golub. Classics in Applied Mathematics, 19. Society for Industrial and Applied Mathematics (SIAM), Philadelphia, PA, (1997).

\section{Cemil Tunc}

Department of Mathematics

Faculty of Science

Yuzuncu Yil University

65080, Van-Turkey

Turkey

e-mail : cemtunc@yahoo.com 